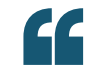

new avenues

that could

be explored

for the novel

therapeutic

intervention

of fungal

infections
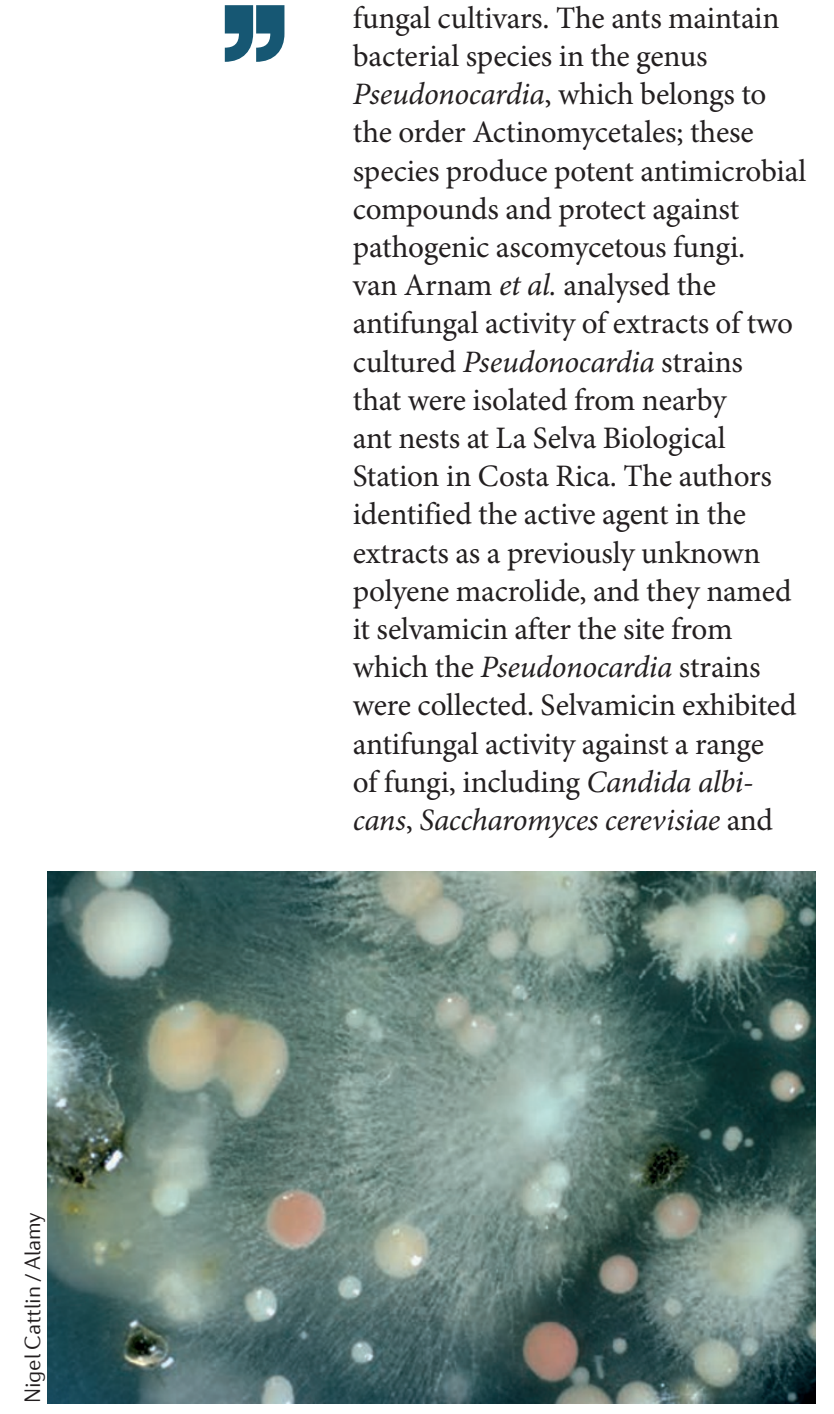

The need for new antifungals is increasing, given the emerging resistance of fungi to current drugs and the toxicity of these drugs to humans. One study now reports the identification of a previously unknown antifungal compound that is produced by ant-associated bacteria, and another study identifies a histone deacetylase as a potential new antifungal target.

Fungus-growing ants exist in a mutualistic relationship with their fungal cultivars. The ants maintain bacterial species in the genus Pseudonocardia, which belongs to the order Actinomycetales; these species produce potent antimicrobial compounds and protect against pathogenic ascomycetous fungi. van Arnam et al. analysed the antifungal activity of extracts of two cultured Pseudonocardia strains that were isolated from nearby ant nests at La Selva Biological Station in Costa Rica. The authors identified the active agent in the extracts as a previously unknown polyene macrolide, and they named it selvamicin after the site from which the Pseudonocardia strains were collected. Selvamicin exhibited antifungal activity against a range of fungi, including Candida albicans, Saccharomyces cerevisiae and

\title{
ANTIFUNGALS
}

\section{Uncovering new drugs and targets}

Aspergillus fumigatus. By contrast, no activity was observed against Gramnegative or Gram-positive bacteria.

Structural studies showed that, although selvamicin had similar features to the antifungal agents amphotericin B and nystatin A1, it also exhibited distinct features, such as a second sugar, a truncated macrocyclic core and a lack of carboxylate and ammonium groups. Furthermore, although the antifungal activity of selvamicin was less than that of nystatin A1, it was more soluble, which is an advantage over currently used polyenes, perhaps owing to its second sugar moiety. Further studies are now required to determine the mechanism of action of this novel antifungal agent.

Finally, the authors identified the selvamicin biosynthetic gene cluster (BGC), which was identical in both Pseudonocardia strains, but, interestingly, was differentially located either on a plasmid or on the chromosome. This, together with the finding that the BGC is flanked by mobile genetic elements, including transposases and integrases, suggests that this gene cluster is likely to be spread through horizontal gene transfer.

In a second study, Bauer, Varadarajan et al. identified the fungal class 1 type histone deacetylase (HDAC) RpdA as a potential antifungal drug target. Previous studies, and this report, failed to generate RPDAnull mutants in several Aspergillus species, which indicates that RpdA is essential for the viability of filamentous fungi. By contrast, the single or multiple deletion of other HDACs only had a moderate effect on fungal growth.

The authors found that RPDA mutants were not viable owing to the lack of HDAC activity, and inhibition of the catalytic activity of RpdA using trichostatin A (TSA; a known potent inhibitor of classic HDACs) decreased the number of germinating spores and the length of hyphae in A. fumigatus and other pathogenic relatives. Vegetative growth was also inhibited by TSA, as strains that were treated with this compound showed growth retardation and disordered mycelia. Thus, TSA exhibits antifungal activity.

Next, Bauer, Varadarajan et al. identified two fungus-specific regions in RpdA: an extension at the amino-terminal end and an acidic motif within the extended carboxyl terminus. A mutant in which the $\mathrm{N}$-terminal region was deleted, but not a mutant that lacked the acidic motif, rescued the growth retardation phenotype of strains in which wild-type RpdA was suppressed. Moreover, the nuclear translocation and catalytic activity of the enzyme were dependent on the presence of the acidic motif within the $\mathrm{C}$ terminus; this motif is missing from homologous proteins in yeasts and higher eukaryotes. Thus, the essential role and fungal-specific features of RpdA suggest that it is a promising target for HDAC inhibitors that have antifungal activity. Indeed, HDAC inhibitors are already approved by the FDA or are under development for cancer therapy, and these advances might facilitate the use of HDAC inhibitors for the treatment of mycosis.

In summary, these studies discover new avenues that could be explored for the novel therapeutic intervention of fungal infections.

Andrea Du Toit

ORIGINAL ARTICLES Van Arnam, E. B. et al. Selvamicin, an atypical antifungal polyene from two alternative genomic contexts. Proc. Natl Acad. Sci. USA http://dx.doi.org/10.1073/ pnas.1613285113 (2016) | Bauer, I., Varadarajan, D. et al. A class 1 histone deacetylase with potential as an antifungal target. $m B i o$ 7, e00831-16 (2016) 